\section{Reply to "A combination treatment approach and cord blood stem cell transplant for blastic plasmacytoid dendritic cell neoplasm" and to "A low-grade B-cell lymphoma with prolymphocytic/paraimmunoblastic proliferation and IRF4 rearrangement" Dendritic cell leukemia}

Two recent reports by Ramanathan et al. ${ }^{1}$ and Hansford et $a l^{2}$ commented on the findings of our paper "Blastic plasmacytoid dendritic cell neoplasm with leukemic presentation: an Italian multicenter study" in which we evaluated the clinical features and the efficacy of treatments in 43 patients at onset of a leukemic form of blastic plasmacytoid dendritic cell neoplasm (BPDCN). Since there is no consensus as to the best therapeutic approach for this rare and aggressive disease, based on 'real life' data, we suggested a strategy of combining acute lymphoid leukemia(ALL)/lymphoma-type and acute myeloid leukemia(AML)-type regimens, emphasizing the importance of performing an allogeneic hemotopoietic stem cell transplant (HSCT) in first complete remission as consolidation strategy, given the poor prognosis of patients (median overall survival 8.7 months). We are grateful for the clinical appraisal of our research and we appreciate the comments from colleagues that allow some further considerations to be made.

Ramanathan et al. ${ }^{1}$ described the case of BPDCN onset in a young woman as a leukemia, achieving complete remission after treatment according to regimens combining ALL-type and AML-type therapy, followed by consolidation with cord blood stem cell transplantation. They agree with us that this is a feasible and effective therapeutic strategy for this rare and aggressive leukemia. We agree with the authors that the cord blood stem transplantation reduced the toxicity of acute graft-versus-host disease (GVHD) in patients whose clinical condition is already severely compromised by the underlying malignancy. Unfortunately, there are no randomized clinical trials supporting the use of ALL-type or AML-type regimens, but some biological factors can offer a rationale for the ALLtype approach. In fact, although plasmacytoid dendritic cells are commonly considered within the myeloid compartment, their derivation is quite complex. In fact, these cells can derive from both myeloid and lymphoid progenitors, with the second option being that preferred during physiological development. It is possible that BPDCN cells may retain some biological features of lymphoid elements, requiring specific therapies. However, more refined molecular analyses are necessary to gain further information about the pathobiology of this disease. This may help identify novel potential therapeutic targets and open new scenarios in the management of the disease. ${ }^{4,5}$

Hansford et al. ${ }^{2}$ report a case of a 2 -year old girl with BPDCN who obtained a complete remission after AML therapy. She is still alive without relapse of disease two years after completion of therapy, without the need for HSCT. These data are in contrast with the results of a recent analysis by Jegalian et al. focusing on pediatric cases. $^{6}$ They demonstrated a quite brilliant efficacy of ALL- type schemes in children affected by BPDCN, allowing the patients to achieve complete remission and with a better prognosis, including long-term survivals, compared to adults. ${ }^{6}$ In fact, the therapeutic schedule used by Hansford et $a l^{2}$ for their 2-year old patient is a protocol for the treatment of AML, but it includes drugs such as L-asparaginase, typically used for the treatment of lymphoblastic leukemia both in children and in adult patients. Furthermore, we know that children have a better prognosis and this could explain their good response to such induction therapy.

We agree with both Ramanathan et al. ${ }^{1}$ and Hansford et $a l^{2}$ in underlining the importance of an extensive immunohistochemical analysis of neoplastic cells before a definitive diagnosis is made.

Finally, the rarity of the disease means that it is difficult to carry out specific clinical trials. We, therefore, agree with Hansford and colleagues that an international registry, including both pediatric and adult cases, is essential in order to widen our knowledge of the disease and of its molecular and biological features. In this way, a standardized evidenced-based therapeutic strategy could be established. It must be recognized, however, that this will not be an easy task and it will take a long time before sufficient data are collected to allow us to draw definitive conclusions.

\section{Livio Pagano, Caterina Giovanna Valentini, and Fabio Facchetti}

IInstitute of Hematology, Catholic University, Rome; ${ }^{2}$ Department of Pathology, University of Brescia, Italy

Correspondence:Ipagano@rm.unicatt.it doi:10.3324/haematol.2013.084582

Key-words: blastic plasmacytoid dendritic cell neoplasm, cord blood transplant, pediatric.

Information on authorship, contributions, and financial \& other disclosures was provided by the authors and is available with the online version of this article at www. haematologica.org.

\section{References}

1. Ramanathan M, Cerny J, Yu H, Woda BA, Nath R. A combination Treatment Approach and Cord Blood Stem Cell Transplant for Blastic Plasmacytoid Dendritic Cell Neoplasm. Haematologica; 2013;98(3):e36

2. Hansford R, Kotecha RS, Jevon G, Gottardo NG. Efficacy of Acute Myeloid Leukemia Therapy without Stem-Cell Transplantation in a child with Blastic Plasmacytoid Dendritic Cell Neoplasm. Haematologica; 2013;98(3):e30-1.

3. Pagano L, Valentini CG, Pulsoni A, Fisogni S, Carluccio P, Mannelli F, et al. Blastic plasmacytoid dendritic cell neoplasm with leukemic presentation: an Italian multicenter study. Haematologica. 2012 Oct 12. [Epub ahead of print] doi:10.3324/haematol.2012.072645.

4. O'Garra A, Trinchieri G. Are dendritic cells afraid of commitment? Nat Immunol. 2004;5(12):1206-8.

5. Piccaluga PP, Paolini S, Sapienza MR, Pileri SA. Blastic plasmacytoid dendritic cell neoplasm: is it time to redefine the standard of care? Expert Rev Hematol. 2012;5(4):353-5.

6. Jegalian AG, Buxbaum NP, Facchetti F, Raffeld M, Pittaluga S, Wayne AS, et al. Blastic plasmacytoid dendritic cell neoplasm in children: diagnostic features and clinical implications. Haematologica. 2010;95(11):1873-9. 